\title{
Primes and Irreducibles in Truncation Integer Parts of Real Closed Fields. *
}

\author{
D. Biljakovic, M. Kochetov and S. Kuhlmann ${ }^{\dagger}$
}

04. 06. 2004

\begin{abstract}
Berarducci (2000) studied irreducible elements of the ring $k\left(\left(G^{<0}\right)\right) \oplus \mathbb{Z}$, which is an integer part of the power series field $k((G))$ where $G$ is an ordered divisible abelian group and $k$ is an ordered field. Pitteloud (2001) proved that some of the irreducible elements constructed by Berarducci are actually prime. Both authors mainly concentrated on the case of archimedean $G$. In this paper, we study truncation integer parts of any (non-archimedean) real closed field and generalize results of Berarducci and Pitteloud. To this end, we study the canonical integer part $\operatorname{Neg}(F) \oplus \mathbb{Z}$ of any truncation closed subfield $F$ of $k((G))$, where $\operatorname{Neg}(F):=F \cap k\left(\left(G^{<0}\right)\right)$, and work out in detail how the general case can be reduced to the case of archimedean $G$. In particular, we prove that $k\left(\left(G^{<0}\right)\right) \oplus \mathbb{Z}$ has (cofinally many) prime elements for any ordered divisible abelian group $G$. Addressing a question in the paper of Berarducci, we show that every truncation integer part of a non-archimedean exponential field has a cofinal set of irreducible elements. Finally, we apply our results to two important classes of exponential fields: exponential algebraic power series and exponential-logarithmic power series.
\end{abstract}

\section{Introduction}

An integer part (IP for short) $Z$ of an ordered field $K$ is a discretely ordered subring, with 1 as the least positive element, and such that for every $x \in K$, there is a $z \in Z$ such that $z \leq x<z+1$. It follows that the ring of integers $\mathbb{Z}$ is a convex subring of $Z$. If $K$ is archimedean, then $\mathbb{Z}$ is the only IP of $K$, so we will be interested in the case of non-archimedean $K$.

*1991 Mathematics Subject Classification: Primary 06F25; Secondary 13A16, 03H15, 03E10, 12J25, $13 \mathrm{~A} 05$.

${ }^{\dagger}$ Second author supported by an NSERC Postdoctoral Fellowship, third author partially supported by an NSERC Discovery Grant. This paper was written while the third author was on sabbatical leave at Université Paris 7. The authors wish to thank the Equipe de Logique de Paris 7 for its support and hospitality. 
Shepherdson [S] showed that IP's of real closed fields are precisely the models of a fragment of Peano Arithmetic called Open Induction (OI for short). OI is the firstorder theory, in the language $L:=\{+, \cdot,<, 0,1\}$, of discretely ordered commutative rings with 1 whose set of non-negative elements satisfies, for each quantifier-free formula $\Phi(x, y)$, the associated induction axiom $I(\Phi)$ :

$$
\forall y[\Phi(0, y) \text { and } \forall x[\Phi(x, y) \rightarrow \Phi(x+1, y)] \rightarrow \forall x \Phi(x, y)] .
$$

This correspondence led Shepherdson to investigate the arithmetic properties of IP's of real closed fields. Given a field $k$ and an ordered abelian group $G$, let us denote by $k((G))$ the field of generalized power series with exponents in $G$ and coefficients in $k$ (see Section 1). We write $k(G):=k\left(t^{g} \mid g \in G\right)$ for the subfield generated by $k$ and by the monomials $t^{g}$. This subfield is the quotient field of the group ring $k[G]$ (which is a domain by the same argument as for the ring of polynomials). If $k$ is an ordered field, then $k((G))$ can be ordered lexicographically. Shepherdson considered the countable recursive "algebraic Puiseux series field" $\mathbb{Q}\left(t^{g} \mid g \in \mathbb{Q}\right)^{r}$, which can be viewed as a subfield of the field $\mathbb{Q}^{r}((\mathbb{Q}))$ of power series with coefficients in the field of real algebraic numbers $\mathbb{Q}^{r}$ and exponents in the additive group of rational numbers $\mathbb{Q}$. (The superscript $r$ denotes real closure.) He constructed an IP of this field in which the only irreducible elements are those in $\mathbb{Z}$. In particular, the set of primes is not cofinal in this model of OI. Thus the "infinity of primes" is not provable from Open Induction. On the other hand, subsequent to the work of Shepherdson, several authors (e.g. $[\mathrm{M}],[\mathrm{B}-\mathrm{O}],[\mathrm{Bi}]$ ) constructed various models of OI with (a cofinal set of) infinite primes.

In $[\mathrm{M}-\mathrm{R}]$, Mourgues and Ressayre establish the existence of an IP for any real closed field $K$ as follows. Let $V$ be the natural valuation on $K$. Denote by $k$ the residue field and by $G$ the value group of $K$ (see Section 1). [M-R] show that there is an order preserving embedding $\varphi$ of $K$ into the field of generalized power series $k((G))$ such that $\varphi(K)$ is a truncation closed subfield (see Section 2 for details). They observe that for the field $k((G))$, an integer part is given by $k\left(\left(G^{<0}\right)\right) \oplus \mathbb{Z}$, where $k\left(\left(G^{<0}\right)\right)$ is the (non-unital) ring of power series with negative support. It follows easily (see Proposition 2.1) that for any truncation closed subfield $F$ of $k((G))$, an integer part is given by $Z_{F}=\operatorname{Neg}(F) \oplus \mathbb{Z}$, where $\operatorname{Neg}(F):=k\left(\left(G^{<0}\right)\right) \cap F$. We shall call $Z_{F}$ the canonical integer part of $F$. Finally $\varphi^{-1}\left(Z_{F}\right)$ is an integer part of $K$ if we take $F=\varphi(K)$. An integer part $Z$ of $K$ obtained in this way from a truncation closed embedding shall be called a truncation integer part of $K$.

In $[R]$, a proof for an exponential analogue of the main result of $[M-R]$ is sketched: every exponential field (see Section 5) has an exponential integer part (EIP for short). An EIP is an IP that satisfies some closure conditions under the exponential function (see Section 5).

In [Bo2], Boughattas considers the following extension of OI in the language $L \cup\left\{2^{x}\right\}$ containing a symbol for the exponential function. He defines $\mathrm{OI}\left(2^{x}\right)$ to be the 
first-order theory of discretely ordered commutative rings with 1 whose set of nonnegative elements satisfies, for each quantifier-free formula $\Phi(x, y)$ of $L \cup\left\{2^{x}\right\}$, the associated induction axiom $I(\Phi)$, and also the following basic axioms for $2^{x}$ :

$$
2^{0}=1, \quad 2^{1}=2, \quad 2^{x+y}=2^{x} 2^{y}, \quad x<2^{x} .
$$

Analogously to Shepherdson's result, Boughattas constructs a model of OI $\left(2^{x}\right)$ in which the only irreducible elements are those in $\mathbb{Z}$, and thus the set of primes is not cofinal in this model of $\mathrm{OI}\left(2^{x}\right)$. We note that, unlike the case of OI, an algebraic description of models of $\mathrm{OI}\left(2^{x}\right)$ is not known. In particular, the relationship between models of $\mathrm{OI}\left(2^{x}\right)$ on the one hand and EIP's of exponential fields on the other hand remains unclear. In [B; Concluding Remarks], Berarducci asks (and attributes the question to Ressayre) for an explicit axiomatisation of the class of EIP's of exponential fields. We do not consider this question in this paper.

Using a new kind of valuation whose values are ordinal numbers, Berarducci [B] studies irreducible elements in the ring $k\left(\left(G^{<0}\right)\right) \oplus \mathbb{Z}$, that is, in the canonical IP of the power series field $k((G))$, focusing mainly on the case when $G$ is archimedean. He gives a test for irreducibility based only on the order type of the support of a series. It is not known if every irreducible element of $k((G))$ is prime (that is, generates a prime ideal). Refining the methods of $[\mathrm{B}]$, Pitteloud $[\mathrm{P}]$ shows that some of the irreducible series constructed in $[\mathrm{B}]$ are actually prime, in the case when $G$ contains a maximal proper convex subgroup.

In this paper, we extend the results of $[\mathrm{B}]$ and $[\mathrm{P}]$ to the canonical integer part $\operatorname{Neg}(F) \oplus \mathbb{Z}$ of any truncation closed subfield $F$,

$$
k(G) \subset F \subset k((G))
$$

for an arbitrary divisible ordered abelian group $G \neq 0$. We shall denote by $k\left[G^{<0}\right]$ (respectively, by $k\left[G^{\leq 0}\right]$ ) the semigroup ring consisting of power series with negative (respectively, non-positive) and finite support. Note that $k\left[G^{<0}\right] \subset \operatorname{Neg}(F)$ since $k(G) \subset F$. [B; Theorem 11.2] says that, in the case of archimedean $G$, all irreducible elements of $k\left[G^{<0}\right] \oplus \mathbb{Z}$ remain irreducible in $k\left(\left(G^{<0}\right)\right) \oplus \mathbb{Z}$. We study, for an arbitrary $G$, the behaviour of primes and irreducibles under the ring extensions

$$
k\left[G^{<0}\right] \oplus \mathbb{Z} \subset \operatorname{Neg}(F) \oplus \mathbb{Z} \subset k\left(\left(G^{<0}\right)\right) \oplus \mathbb{Z}
$$

In [B; Concluding Remarks], the author asks whether every EIP of an exponential field contains a cofinal set of irreducible elements. We give a partial answer to this question: applying our results to truncation EIP's of non-archimedean exponential fields, we show that these EIP's indeed contain a cofinal set of irreducible elements. Note that in this case, the rank of $G$ (see Section 2) is a dense linearly ordered set without endpoints [K ; Corollary 1.23], in particular $G$ cannot be archimedean, nor can it contain a maximal proper convex subgroup. 
The structure of this paper is the following. In Section 1, we fix the notation and review the necessary background concerning ordered groups and fields. In Section 2, we establish some straightforward facts used in the subsequent sections, in particular, to study the extensions (1). The main results of Section 3 are Proposition 3.8 and Corollary 3.10 which provides (under mild conditions) cofinal sets of irreducibles (=primes) in $k\left[G^{<0}\right] \oplus \mathbb{Z}$.

In Section 4, we generalize [B; Theorem 11.2] (see Corollaries 4.4 and 4.5) using the reduction to the case of an archimedean group $G$ given in our Theorem 4.2 and Corollary 4.3 (some special cases of Corollary 4.3 already appeared in [B] and $[\mathrm{P}])$. As a consequence, we extend Corollary 3.10 to the ring $k\left(\left(G^{<0}\right)\right) \oplus \mathbb{Z}$ obtaining cofinal sets of irreducibles with finite support in $\operatorname{Neg}(F) \oplus \mathbb{Z}$ (see Corollary 4.10). In the special case when $F$ is the field of algebraic power series, that is, $F=k(G)^{r}$, we can improve the result to obtain cofinal sets of primes with finite support in $\operatorname{Neg}(F) \oplus \mathbb{Z}$ (see Corollary 4.11). Using our generalization of [P; Theorem 4.2] (see Theorem 4.12), we show that $k\left(\left(G^{<0}\right)\right) \oplus \mathbb{Z}$ has a cofinal set of primes with infinite support (see Corollary 4.16).

In Section 5, we apply the results of Section 4 to study EIP's of exponential fields. The main application is given in Theorem 5.3: we establish that every exponential field has an EIP with a cofinal set of irreducible elements. We work out two examples in detail. In Example 5.4, we consider the countable "Algebraic Power Series Fields with Exponentiation" described in [K; Example 1.45]. These fields are of the form $E\left(t^{g} \mid g \in G\right)^{r}$, where $E$ is a countable exponentially closed subfield of the reals, and $G$ is the lexicographic sum, taken over the rationals, of copies of the additive group of $E$. These fields may be viewed as truncation closed subfields of $E((G))$. We start by studying the canonical IP of such a field and show that this canonical IP is an EIP. We establish that this canonical EIP has a cofinal set of prime elements with finite support. In Example 5.5 we study the canonical EIP of the "Exponential-Logarithmic Power Series Fields" introduced in [K; Chapter 5 p. 79]. We show that this EIP has cofinally many primes with infinite support.

\section{Preliminaries.}

Let $G$ be an ordered abelian group. Set $|g|:=\max \{g,-g\}$ for $g \in G$. For non-zero $g_{1}, g_{2} \in G$ we say that $g_{1}$ is archimedean equivalent to $g_{2}$ if there exists $r \in \mathbb{N}$ such that

$$
r\left|g_{1}\right| \geq\left|g_{2}\right| \text { and } r\left|g_{2}\right| \geq\left|g_{1}\right| \text {. }
$$

We write $g_{1}<<g_{2}$ if $r\left|g_{1}\right|<\left|g_{2}\right|$ for all $r \in \mathbb{N}$. Denote by $[g]$ the equivalence class of $g \neq 0$, and by $v$ the natural valuation on $G$, that is, $v(g):=[g]$ for $g \neq 0$, and $v(0):=\infty$. The rank of $G$ is defined to be $\Gamma:=v(G \backslash\{0\})$. The relation $<<$ on $G$ induces a linear order on the set $\Gamma$. For each $\gamma \in \Gamma$, fix $g_{\gamma} \in G$ such that $v\left(g_{\gamma}\right)=\gamma$. Let $C_{\gamma}$ and $D_{\gamma}$ denote, respectively, the smallest convex subgroup 
containing $g_{\gamma}$ and the largest convex subgroup not containing $g_{\gamma}$. Note that $C_{\gamma}$ and $D_{\gamma}$ are independent from the choice of the representative $g_{\gamma}$. In fact

$$
C_{\gamma}=\{g \in G \mid v(g) \geq \gamma\} \text { and } D_{\gamma}=\{g \in G \mid v(g)>\gamma\} \text {. }
$$

Note also that $D_{\gamma}$ is a maximal proper convex subgroup of $C_{\gamma}$ and thus the quotient

$$
A_{\gamma}:=C_{\gamma} / D_{\gamma}
$$

is archimedean. We call $A_{\gamma}$ an archimedean component of $G$.

Let $Q$ be an archimedean field. We now recall some general definitions and facts about ordered $Q$-vector spaces. Clearly, if $G$ is an ordered $Q$-vector space, then for $g_{1}, g_{2} \in G, g_{1}$ is archimedean equivalent to $g_{2}$ if and only if there exists $r \in Q$ such that (2) holds. A subset $B \subset G \backslash\{0\}$ is strongly independent if $B$ consists of pairwise archimedean inequivalent elements. We say that $B=\left\{b_{i} \mid i \in I\right\}$ is $Q$-valuation independent if for all $r_{i} \in Q$ such that $r_{i}=0$ for all but finitely many $i \in I$,

$$
v\left(\sum_{i \in I} r_{i} b_{i}\right)=\min _{\left\{i \in I \mid r_{i} \neq 0\right\}}\left\{v\left(b_{i}\right)\right\} .
$$

A $Q$-valuation basis is a $Q$-basis which is $Q$-valuation independent.

If $G$ is a $Q$-vector space, then $C_{\gamma}$ and $D_{\gamma}$ are $Q$-subspaces, so $A_{\gamma}$ is a $Q$-vector space, isomorphic to any maximal archimedean subspace of $G$ containing $g_{\gamma}$, and to any $Q$-vector space complement to $D_{\gamma}$ in $C_{\gamma}$.

Remark 1.1 For $Q=\mathbb{Q}, A_{\gamma}$ is isomorphic to any maximal archimedean subgroup of $G$ containing $g_{\gamma}$. This is because if $A$ is a subgroup of $G$, then $A$ is archimedean if and only if the divisible hull $\langle A\rangle_{\mathbb{Q}}$ of $A$ is archimedean, which implies that a maximal archimedean subgroup of $G$ is necessarily a $\mathbb{Q}$-subspace.

We let $\pi_{\gamma}$ denote the natural homomorphism $C_{\gamma} \rightarrow A_{\gamma}=C_{\gamma} / D_{\gamma}$. The following characterization of valuation independence is useful and easy to prove (see $[\mathrm{K}]$ ).

Proposition 1.2 Let $B \subset G \backslash\{0\}$. Then $B$ is $Q$-valuation independent if and only if the following holds: for all $n \in \mathbb{N}$ and distinct $b_{1}, \ldots, b_{n} \in B$ with $v\left(b_{1}\right)=$ $\ldots=v\left(b_{n}\right)=\gamma$, the elements $\pi_{\gamma}\left(b_{1}\right), \ldots, \pi_{\gamma}\left(b_{n}\right)$ in $A_{\gamma}$ are $Q$-linearly independent.

It follows that a $Q$-valuation basis is a maximal $Q$-valuation independent set. (But in general, a maximal $Q$-valuation independent set need not be a basis.) We also have

Corollary 1.3 Let $B \subset G$ be $Q$-linearly independent and consist of archimedean equivalent elements. Then $\langle B\rangle_{Q}$ is archimedean if and only if $B$ is $Q$-valuation independent. 
If all archimedean components of $G$ have dimension 1 over $Q$, then $B \subset G$ is $Q$ valuation independent if and only if $B$ is strongly independent. (In particular, if $Q=\mathbb{R}$, then $B$ is $Q$-valuation independent if and only if $B$ is strongly independent, because an archimedean $\mathbb{R}$-vector space has necessarily dimension 1 over $\mathbb{R}$.)

In $[\mathrm{Br}]$ it is shown that every ordered $Q$-vector space of countable dimension has a $Q$-valuation basis. (In particular, every ordered $\mathbb{R}$-vector space of countable dimension has a strongly independent basis.)

We also need to recall some facts about valued fields. (In this paper, we mainly deal with fields of characterisitc 0 .) Let $K$ be a field, $G$ an ordered abelian group and $\infty$ an element greater than every element of $G$.

A surjective map $w: K \rightarrow G \cup\{\infty\}$ is a valuation on $K$ if for all $a, b \in K$

(i) $w(a)=\infty$ iff $a=0$, (ii) $w(a b)=w(a)+w(b)$, (iii) $w(a-b) \geq \min \{w(a), w(b)\}$.

We say that $(K, w)$ is a valued field. The value group of $(K, w)$ is $w(K):=G$. The valuation ring of $w$ is $\mathcal{O}_{w}:=\{a \mid a \in K$ and $w(a) \geq 0\}$ and the valuation ideal is $\mathcal{M}_{w}:=\{a \mid a \in K$ and $w(a)>0\}$. The field $\mathcal{O}_{w} / \mathcal{M}_{w}$, denoted by $K w$, is the residue field. For $b \in \mathcal{O}_{w}$, bw is its image under the residue map.

A valued field $(K, w)$ is henselian if given a polynomial $p(x) \in \mathcal{O}_{w}[x]$, and $a \in K w$ a simple root of the reduced polynomial $p(x) w \in K w[x]$, we can find a root $b \in K$ of $p(x)$ such that $b w=a$.

Let $K \subset L$ be an extension of valued fields. By abuse of notation, denote by $w$ the valuation on both $K$ and $L$. Then $K$ is said to be $w$-dense in $L$ if for every $a \in L$ and $\alpha \in w(L)$ there is some $b \in K$ such that $w(a-b)>\alpha$.

If $(K,+, \cdot, 0,1,<)$ is an ordered field, we denote by $V$ its natural valuation, that is, the natural valuation $V$ on the ordered abelian group $(K,+, 0,<)$. (The set of archimedean classes becomes an ordered abelian group by setting $[x]+[y]:=[x y]$.) For the natural valuation, we shall use the notation $\mathcal{O}_{K}:=\{x \in K \mid V(x) \geq 0\}$ and $\mathcal{M}_{K}:=\{x \in K \mid V(x)>0\}$, respectively, for the valuation ring and valuation ideal. We shall also often write $k$ for the residue field $\mathcal{O}_{K} / \mathcal{M}_{K}$ and $G$ for the value group $V(K)$. Note that $k$ is an archimedean ordered field, and that $V$ is compatible with the order, that is, has a convex valuation ring. We denote by $\mathcal{U}_{K}^{>0}$ the multiplicative group of positive units (invertible elements) of $\mathcal{O}_{K}$. The subgroup $1+\mathcal{M}_{K}$ of $\mathcal{U}_{K}^{>0}$ is called the group of 1-units.

For ordered fields, there is another notion of density that we would like to review briefly. For more details see [K; Chapter 1, Section 6]. Let $K \subset L$ be an extension of ordered fields. $K$ is order dense in $L$ if for all $a, c \in L$ with $a<c$ there is $b \in K$ such that $a \leq b \leq c$. Let $K \subset L$ be an extension of ordered fields, and let $w$ be a compatible valuation on both of them. Assume that $L$ is non-archimedean. Then $K$ is order dense in $L$ if and only if $K$ is $w$-dense in $L$.

We need further well-known facts about ordered fields endowed with a compatible valuation. Let $K$ be an ordered field and $w$ a compatible valuation on $K$. Then 
$K$ is real closed if and only if (i) $(K, w)$ is henselian (ii) its residue field $K w$ is real closed field and (iii) its value group $w(K)$ is divisible.

If $K$ is real closed, then the residue field $K w$ embeds in $K$ and $K$ admits a crosssection, that is, an embedding $\eta$ of the value group $G$ into $\left(K^{>0}, \cdot\right)$ such that $w(\eta(g))=g$ for all $g \in G$ (see $[\mathrm{PC}])$.

Therefore, whenever needed, we can assume without loss of generality that $k(G) \subset$ $K$. (More precisely, we identify the residue field $k$ with a maximal subfield of $\mathcal{O}_{K}$ through the residue map, and $G$ with $\eta(G)$.)

Recall from the Introduction that for $G$ an ordered abelian group, $k$ a field, $k((G))$ denotes the field of power series with coefficients in $k$ and exponents in $G$. Every series $s \in k((G))$ is of the form $\sum_{g \in G} s_{g} t^{g}$ with $s_{g} \in k$ and well-ordered support $\left\{g \in G \mid s_{g} \neq 0\right\}$. Addition is pointwise, multiplication is given by the usual formula for multiplying power series:

$$
\left(\sum_{g \in G} r_{g} t^{g}\right)\left(\sum_{g \in G} s_{g} t^{g}\right)=\sum_{g \in G}\left(\sum_{g^{\prime} \in G} r_{g^{\prime}} s_{g-g^{\prime}}\right) t^{g} .
$$

The canonical valuation on $k((G))$ is given by $V_{\min }(s):=\min (\operatorname{support} s)$ for any series $s \in k((G))$. If $k$ is an ordered field, we can endow $k((G))$ with the lexicographic order: a series is positive if its least nonzero coefficient is positive. With this order, $k((G))$ is an ordered field, and $V_{\min }$ is compatible with this order. If $k$ is archimedean, then $V_{\min }$ coincides with the natural valuation $V$. Clearly, the value group of $\left(k((G)), V_{\min }\right)$ is (isomorphic to) $G$ and the residue field is (isomorphic to) $k$. The valuation $\operatorname{ring} k\left(\left(G^{\geq 0}\right)\right)$ consists of the series with non-negative exponents, and the valuation ideal $k\left(\left(G^{>0}\right)\right)$ of the series with positive exponents. The constant term of a series $\sum_{g \in G} s_{g} t^{g}$ is the coefficient $s_{0}$. The units of $k\left(\left(G^{\geq 0}\right)\right)$ are the series in $k\left(\left(G^{\geq 0}\right)\right)$ with a non-zero constant term.

Every series $s \in k((G))$ can be written as $s=s_{<0}+s_{0}+s_{>0}$ where $s_{0}$ is the constant term of $s$ and $s_{<0}$, resp. $s_{>0}$, denotes the restriction of $s$ to $G^{<0}$, resp. $G^{>0}$. Thus the (non-unital) ring $k\left(\left(G^{<0}\right)\right)$ of generalized power series with negative support is a complement in $(k((G)),+)$ to the valuation ring. Note that it is in fact a $k$-algebra. We shall denote by $k\left(\left(G^{\leq 0}\right)\right)$ the ring of generalized power series with non-positive support.

Given $s \in k((G))^{>0}$, we can factor out the monomial of smallest exponent $g \in$ $G$ and write $s=t^{g} u$ with $u$ a unit with a positive constant term. Thus the multiplicative subgroup Mon $k((G)):=\left\{t^{g} \mid g \in G\right\}$ consisting of the (monic) monomials $t^{g}$ is a complement in $\left(k((G))^{>0}, \cdot\right)$ to the subgroup of positive units.

\section{Truncation Integer Parts.}

A subfield $F$ of $k((G))$ is truncation closed if whenever $s \in F$, the restriction of $s$ to any initial segment of $G$ also belongs to $F$. As mentioned in the Introduction, 
[M-R] show that given a real closed field $K$ with residue field $k$ and value group $G$ (for the natural valuation $V$ on $K$ ), with $k(G) \subset K$, there is a truncation closed embedding of $K$ into $k((G))$ over $k(G)$, that is, an embedding $\varphi$ such that $F:=\varphi(K)$ is truncation closed. Note that since the restriction of $\varphi$ to $k(G)$ is the identity, $\varphi$ is in particular an embedding of $k$-vector spaces.

Proposition 2.1 Let $F \subset k((G))$ be a truncation closed subfield that contains $k(G)$. Then $Z_{F}:=\operatorname{Neg}(F) \oplus \mathbb{Z}$ is an integer part of $F$ (that contains $k\left[G^{<0}\right] \oplus \mathbb{Z}$ ).

Proof: $\quad$ Clearly, $Z_{F}$ is a discrete subring of $F$. Let $s \in k((G))$. Let $\left\lfloor s_{0}\right\rfloor \in \mathbb{Z}$ be the integer part of $s_{0} \in k$. Define

$$
z_{s}= \begin{cases}s_{<0}+s_{0}-1 & \text { if } s_{0} \in \mathbb{Z} \text { and } s_{>0}<0, \\ s_{<0}+\left\lfloor s_{0}\right\rfloor & \text { otherwise. }\end{cases}
$$

Clearly, $z_{s} \leq s<z_{s}+1$. From truncation closedness of $F$ it follows that if $s \in F$, then $z_{s} \in Z_{F}$.

It follows that $\varphi^{-1}\left(Z_{F}\right)$ is an IP of $K$. Recall that we refer to IP's obtained in this way via a truncation closed embedding as truncation IP's.

We now state some easy facts about IP's of ordered fields in general. We note that all IP's of a given ordered field are isomorphic as ordered sets (if $Z_{1}, Z_{2}$ are IP's, an isomorphism is obtained by mapping $s \in Z_{1}$ to its integer part $z_{s}$ with respect to $Z_{2}$ ), but in general, they need not be isomorphic as ordered rings, not even elementarily equivalent (see Remark 2.3). If $Z$ is an IP of $K$, then the fraction field of $Z$ is an order-dense subfield of $K$. If $L$ is an order-dense subfield of $K$, then every IP of $L$ is an IP of $K$. Conversely, if $L \subset K$ and there exists an IP $Z$ of $L$ that remains an IP of $K$, then $L$ is order-dense in $K$.

Remark 2.2 In [Bo] examples of ordered fields without IP's are given. We conjectured that every henselian ordered field admits a truncation closed embedding into a field of power series and thus, admits an IP. This conjecture is being studied in $[F]$. We also asked for a direct proof of the existence of IP's for henselian fields (without arguing via truncation closed embeddings in fields of power series). This question is addressed in $[\mathrm{KF}]$.

Remark 2.3 Truncation IP's of non-archimedean real closed fields are very peculiar models of OI. It would be interesting to investigate the algebraic and modeltheoretic properties of this class.

(i) They admit $\mathbb{Z}$ as a direct summand. This is not the case for an arbitrary model of OI. For example, as observed by D. Marker (unpublished), $\mathbb{Z}$ cannot be a direct summand of a non-standard model of Peano Arithmetic. This remark implies in particular that not every IP of a real closed field is a truncation IP.

(ii) They are intimately related to complements of the valuation ring (see $[\mathrm{KF}]$ ). A truncation IP $Z$ of $K$ decomposes as $Z=A \oplus \mathbb{Z}$ (lexicographic sum), where the 
summand $A$ is a $k$-algebra. It is easily verified that $A$ is an additive complement to $\mathcal{O}_{K}$. Since additive complements to $\mathcal{O}_{K}$ are unique up to isomorphism of ordered groups, all truncation IP's of $K$ are isomorphic as ordered groups. Note that if an additive complement $A$ to $\mathcal{O}_{K}$ is closed under multiplication, then $Z(A):=A \oplus \mathbb{Z}$ is an IP of $K$. However, $A$ need not be a $k$-algebra, so $Z(A)$ need not be a truncation IP. It is not known to us whether every IP of the form $Z(A)$, with $A$ a $k$-algebra, is a truncation IP.

(iii) They are never normal (that is, never integrally closed in their field of fractions). This is because $\operatorname{Neg}(F)$ is a $k$-vector space, so $\sqrt{2} t^{g} \in Z_{F}$ for $g \in G^{<0}$ and hence $\sqrt{2}$ is rational over $Z_{F}: \sqrt{2}=\frac{\sqrt{2} t^{g}}{t^{g}}$. Since the normal model obtained in $[\mathrm{B}-\mathrm{O}]$ is an IP of a real closed field, which also has a non-normal IP, we see that the IP's of a given real closed field need not be elementarily equivalent. This motivates the following

Open Question: Does every real closed field have a normal integer part?

Let $F=k(G)$ and $F^{r}$ its real closure. By $[\mathrm{M}-\mathrm{R}], F$ and $F^{r}$ are truncation closed subfields of $k((G))$. We already noted that $k\left[G^{<0}\right] \subset \operatorname{Neg}(F)$. We are interested in understanding when $k\left[G^{<0}\right]=\operatorname{Neg}(F)$. The following fact was observed by F.-V. Kuhlmann.

Proposition 2.4 Assume that $G$ is archimedean and divisible, and that $k$ is a real closed field. Then $\operatorname{Neg}(F)=\operatorname{Neg}\left(F^{r}\right)=k\left[G^{<0}\right]$.

Proof: We first show that $\operatorname{Neg}(F)=k\left[G^{<0}\right]$. Let $f \in F=k(G)$. So $f=p / q$ with $p=\sum_{i=0}^{l} a_{i} t^{g_{i}}, q=\sum_{i=0}^{l} a_{i}^{\prime} t^{g_{i}^{\prime}} \in k[G]$. By factoring out the monomial of least exponent in $q$, say $a_{0}^{\prime} t^{g_{0}^{\prime}}$, we may rewrite

$$
f=\sum_{i=0}^{l} c_{i} t^{h_{i}}(1+\varepsilon)^{-1}=\sum_{i=0}^{l} c_{i} t^{h_{i}} \sum_{n=0}^{\infty}(-1)^{n} \varepsilon^{n}
$$

where $c_{i}=a_{i} / a_{0}^{\prime}, h_{i}=g_{i}-g_{0}^{\prime}$, and $\varepsilon \in k\left[G^{>0}\right]$. Since $G$ is archimedean, we may choose $N$ large enough so that $N V_{\min }(\varepsilon) \geq-h_{i}$ for all $i=0, \ldots, l$. We now rewrite:

$$
f=\sum_{i=0}^{l} c_{i} t^{h_{i}} \sum_{n=0}^{N-1}(-1)^{n} \varepsilon^{n}+\sum_{i=0}^{l} c_{i} t^{h_{i}} \sum_{n=N}^{\infty}(-1)^{n} \varepsilon^{n} .
$$

By our choice of $N$, we have that $V_{\min }\left(\sum_{i=0}^{l} c_{i} t^{h_{i}} \sum_{n=N}^{\infty}(-1)^{n} \varepsilon^{n}\right) \geq 0$. It follows that if $f \in \operatorname{Neg}(F)$, then $f=\sum_{i=0}^{l} c_{i} t^{h_{i}} \sum_{n=0}^{N-1}(-1)^{n} \varepsilon^{n}$, so $f \in k[G]$.

We now show that $\operatorname{Neg}(F)=\operatorname{Neg}\left(F^{r}\right)$. By assumptions on $k$ and $G$, we have $F^{r}=F^{h}:=$ the henselization of $F$ with respect to $V_{\text {min }}$. Since $G$ is archimedean, $F$ is dense in $F^{h}$ (see $[\mathrm{Ri}]$ ). Thus $\operatorname{Neg}(F)=\operatorname{Neg}\left(F^{h}\right)$ (see [K; Lemma 1.32]). 
Remark 2.5 If $G$ is not archimedean, then $k\left[G^{<0}\right] \neq \operatorname{Neg}(F)$ : choose $g_{1}, g_{2} \in G^{<0}$ with $v\left(g_{1}\right)<v\left(g_{2}\right)$, then $t^{g_{1}}\left(1-t^{-g_{2}}\right)^{-1} \in F$ and $t^{g_{1}}\left(1-t^{-g_{2}}\right)^{-1}=\sum_{n=0}^{\infty} t^{g_{1}-n g_{2}}$ has infinite negative support. Also, $\operatorname{Neg}\left(F^{r}\right) \neq \operatorname{Neg}(F)$ : consider $t^{g_{1}}\left(1+t^{-g_{2}}\right)^{1 / 2} \in F^{r}$. Since $t^{g_{1}}\left(1+t^{-g_{2}}\right)^{1 / 2}=t^{g_{1}}+\frac{1}{2} t^{g_{1}-g_{2}}-\frac{1}{8} t^{g_{1}-2 g_{2}}+\cdots$ has negative support, it belongs to $\operatorname{Neg}\left(F^{r}\right)$. On the other hand, $\left(1+t^{-g_{2}}\right)^{1 / 2} \notin F$ so $t^{g_{1}}\left(1+t^{-g_{2}}\right)^{1 / 2} \notin F$.

We will frequently use the following two lemmas. If $R$ is a ring, we denote by $U(R)$ its group of units.

Lemma 2.6 Let $G$ be an ordered abelian group, $K$ a field. Let $R$ be a subring of $K$ and Neg a (non-unital) $K$-subalgebra of $K\left(\left(G^{<0}\right)\right)$. Let $r \in R$. Then $r$ is prime (resp., irreducible) in $R$ iff $r$ is prime (resp., irreducible) in Neg $\oplus R$.

Proof: $\quad$ Clearly, if $a, b \in \operatorname{Neg} \oplus R$ and $a b \in R$, then $a, b \in R$. It follows that $U($ Neg $\oplus R)=U(R)$ and that $r$ is irreducible in $R$ iff $r$ is irreducible in Neg $\oplus R$. Now let $a \in \operatorname{Neg} \oplus R$ and let $a_{0}$ be the constant term of $a$. Clearly, $r$ divides $a$ in Neg $\oplus R$ iff $r$ divides $a_{0}$ in $R$. It follows that $r$ is prime in $R$ iff $r$ is prime in Neg $\oplus R$.

Lemma 2.7 Let $G$ be an ordered abelian group, $K$ a field. Let $R$ be a subring of $K$ and Neg a (non-unital) $K$-subalgebra of $K\left(\left(G^{<0}\right)\right)$. Let $f$ be a non-constant element of $\mathrm{Neg} \oplus R$ with constant term 1 . Then $f$ is prime (resp., irreducible) in Neg $\oplus R$ iff $f$ is prime (resp., irreducible) in Neg $\oplus K$.

Proof: $\quad(\Rightarrow)$ Suppose $f$ is irreducible in Neg $\oplus R$ and assume that $f=a b$ for some non-invertible $a, b \in \mathrm{Neg} \oplus K$. Let $a_{0}, b_{0}$ be the constant terms of $a, b$. Then $a \neq a_{0}, b \neq b_{0}$, and $a_{0} b_{0}=1$. Let $\tilde{a}=b_{0} a$ and $\tilde{b}=a_{0} b$. Then $\tilde{a}, \tilde{b}$ are non-invertible elements of Neg $\oplus R$ and $f=\tilde{a} \tilde{b}$, a contradiction. Now suppose $f$ is prime in Neg $\oplus R, a, b \in \operatorname{Neg} \oplus K$, and $f$ divides $a b$ in Neg $\oplus K$. Let $\tilde{a}=a / a_{0}$ if $a_{0} \neq 0$ and $\tilde{a}=a$ otherwise. Similarly, let $\tilde{b}=b / b_{0}$ if $b_{0} \neq 0$ and $\tilde{b}=b$ otherwise. Then $f$ divides $\tilde{a} \tilde{b}$ in Neg $\oplus R$. Hence $f$ divides $\tilde{a}$ or $\tilde{b}$ in Neg $\oplus R$, which implies that $f$ divides $a$ or $b$ in $\mathrm{Neg} \oplus K$.

$(\Leftarrow)$ Suppose $f$ is irreducible in Neg $\oplus K$ and assume that $f=a b$ for some non-invertible $a, b \in \operatorname{Neg} \oplus R$. Then $a_{0} b_{0}=1$ and, therefore, $a \neq a_{0}$ and $b \neq b_{0}$. But then $a, b$ are not invertible in Neg $\oplus K$ and $f=a b$, a contradiction. Now suppose $f$ is prime in Neg $\oplus K, a, b \in \operatorname{Neg} \oplus R$, and $f$ divides $a b$ in Neg $\oplus R$. Then $f$ divides $a b$ in Neg $\oplus K$. Hence $f$ divides $a$ or $b$ in Neg $\oplus K$, which implies that $f$ divides $a$ or $b$ in Neg $\oplus R$ since $f$ has constant term 1 .

We want to study prime and irreducible elements of the rings $k\left[G^{<0}\right] \oplus \mathbb{Z}$ and $k\left(\left(G^{<0}\right)\right) \oplus \mathbb{Z}$, but it is often more convenient to work with $k\left[G^{\leq 0}\right]$ and $k\left(\left(G^{\leq 0}\right)\right)$ instead. It is easy to see how this change affects primality and irreducibility.

If $f$ is irreducible in $k\left[G^{<0}\right] \oplus \mathbb{Z}$, then either $f \in \mathbb{Z}$ or $f \notin \mathbb{Z}$ and the constant term $f_{0}= \pm 1$. Indeed, if $f \notin \mathbb{Z}$ and $f_{0} \neq \pm 1$, then we can factor $f$ in $k\left[G^{<0}\right] \oplus \mathbb{Z}$ as follows: $f=f_{0}\left(f / f_{0}\right)$ if $f_{0} \neq 0$ and $f=2(f / 2)$ if $f_{0}=0$. Using Lemmas 2.6 
and 2.7, we conclude that prime (resp., irreducible) elements of $k\left[G^{<0}\right] \oplus \mathbb{Z}$ are of two types: 1) primes in $\mathbb{Z}$ and 2) prime (resp., irreducible) elements of $k\left[G^{\leq 0}\right]$ with constant term \pm 1 . We will show in Section 3 that in fact all irreducible elements of $k[G \leq 0]$ are prime.

By similar considerations, primes (resp., irreducibles) of $k\left(\left(G^{<0}\right)\right) \oplus \mathbb{Z}$ are of two types: 1) primes in $\mathbb{Z}$ and 2) primes (resp., irreducibles) of $k\left(\left(G^{\leq 0}\right)\right)$ with constant term \pm 1 . We do not know whether or not all irreducibles of $k\left(\left(G^{\leq 0}\right)\right)$ are prime.

The following two lemmas are standard. We omit the proofs.

Lemma 2.8 Let $(I, \leq)$ be a directed set (that is, $\leq$ is a partial order and, for any $i_{1}, i_{2} \in I$, there exists $i_{0} \in I$ such that $\left.i_{0}>i_{1}, i_{2}\right)$. Let $R$ be an integral domain and assume that, for every $i \in I$, we assigned a subring $R_{i} \subset R$ in such a way that $R$ is the direct limit of $R_{i}$ (that is, $R_{i_{1}} \subset R_{i_{2}}$ for $i_{1} \leq i_{2}$ and $R=\cup_{i \in I} R_{i}$ ). Let $r \in R$. If there exists $i_{0} \in I$ such that $r$ is prime (resp., irreducible) as an element of $R_{i}$ for $i \geq i_{0}$, then $r$ is prime (resp., irreducible) in $R$.

Lemma 2.9 Let $R$ be an integral domain and $T \subset R \backslash\{0\}$ a multiplicative subsemigroup. Denote by $R_{T}$ the corresponding ring of fractions. Let $r \in R$.

1) If $r$ is prime in $R$ and $r \nmid t$ for all $t \in T$, then $r$ is prime in $R_{T}$.

2) If $r$ is prime in $R_{T}$ and, for all $t \in T$ and $a \in R, r|t a \Rightarrow r| a$, then $r$ is prime in $R$.

\section{Primes and Irreducibles in $k\left[G^{\leq 0}\right]$.}

Let $G$ be a torsion-free abelian group and $k$ a field. Then $G$ is orderable, so the group algebra $k[G]$ is a domain. First, we want to show that all irreducibles of $k[G]$ or $k\left[G^{\leq 0}\right]$ are prime.

Proposition 3.1 Let $G$ be a torsion-free abelian group, $k$ a field. Then every irreducible element of $k[G]$ is prime.

Proof: $\quad$ Since $G$ is orderable, $R:=k[G]$ is a domain and the only invertible elements of $R$ are of the form $\lambda t^{g}$ where $g \in G, 0 \neq \lambda \in k$. Let $I$ be the set of finitely generated subgroups of $G$, partially ordered by inclusion. For each $A \in I$, set $R_{A}=k[A]$. Then $R$ is the direct limit of $R_{A}$. Fix an irreducible element $f \in R$. Suppose that $f \in R_{A}$ for some $A \in I$. Since $U\left(R_{A}\right)=U(R) \cap R_{A}, f$ is irreducible in $R_{A}$. Pick a $\mathbb{Z}$-basis $\left\{g_{1}, \ldots, g_{n}\right\}$ of $A$. Then $R_{A} \cong k\left[x_{1}^{ \pm 1}, \ldots, x_{n}^{ \pm 1}\right]$, the ring of Laurent polynomials, where $x_{l}=t^{g_{l}}, l=1, \ldots, n$, so $R_{A}$ is a UFD. It follows that $f$ is prime in $R_{A}$. Applying Lemma 2.8, we conclude that $f$ is prime in $R$.

Corollary 3.2 Let $G$ be an ordered abelian group, $k$ a field. Then every irreducible element of $k\left[G^{\leq 0}\right]$ is prime. 
Proof: Let $f \in k\left[G^{\leq 0}\right]$ be irreducible. Then either $f$ has a nonzero constant term or else $f=\lambda t^{g_{0}}$ where $0 \neq \lambda \in k$ and $g_{0}=\max G^{<0}$. In the second case, $k\left[G^{\leq 0}\right] /(f) \cong k$, so $f$ is prime. In the first case, using the fact that every element $0 \neq s \in k[G]$ can be written uniquely in the form $\tilde{s} t^{g}$ where $g \in G$ and $\tilde{s} \in k\left[G^{\leq 0}\right]$ with nonzero constant term, one checks that $f$ is irreducible in $k[G]$. Then by Proposition 3.1, $f$ is prime in $k[G]$. Applying Lemma 2.9, 2) (with $R:=k\left[G^{\leq 0}\right]$ and $T:=\left\{t^{g} \mid g \in G^{\leq 0}\right\}$ ), we conclude that $f$ is prime in $k\left[G^{\leq 0}\right]$.

Remark 3.3 Note, however, that $k\left[G^{\leq 0}\right]$ and $k[G]$ are rarely UFD's. For instance, if $g \in G^{<0}$ is divisible in $G$ (that is, the equation $n x=g$ has a solution for every $n \in \mathbb{N}$ ), then obviously the (non-invertible) element $t^{g}$ cannot have a factorization into primes in $k\left[G^{\leq 0}\right]$. As to the group ring $k[G]$, if $g \neq 0$ is divisible in $G$ and $k$ is real closed, then the (non-invertible) element $t^{g}+1$ does not have a factorization into primes in $k[G]$. Indeed, assume $t^{g}+1=p_{1} \cdots p_{l}$ is such a factorization. Let $H=\langle g\rangle_{\mathbb{Q}}$, the divisible hull of $\langle g\rangle$, and pick a complement $H^{\prime}$ for $H$ in $G$. Then $k[G]=R\left[H^{\prime}\right]$ where $R=k[H]$. Since $t^{g}+1 \in R$, all $p_{i} \in R$. Since $U(R)=U(k[G]) \cap R, p_{i}$ are irreducible (=prime) in $R$. But this is a contradiction by Proposition 3.5, below.

The following simple observation will be useful:

Lemma 3.4 Let $H \subset G$ be a divisible subgroup and $f \in k[G]$ (resp., $f \in k\left[G^{\leq 0}\right]$ ). Assume that support $f \subset H$. Then $f$ is prime in $k[G]$ (resp., in $k\left[G^{\leq 0}\right]$ ) iff $f$ is prime in $k[H]$ (resp., in $k\left[H^{\leq 0}\right]$ ).

Proof: $\quad$ Pick a complement $H^{\prime}$ for $H$ in $G$. Let $R=k[H] /(f)$. Then $k[G] /(f) \cong$ $R\left[H^{\prime}\right]$. Obviously, $R\left[H^{\prime}\right]$ is a domain iff $R$ is a domain. This proves that $f$ is prime in $k[G]$ iff it is prime in $k[H]$. The case of $k\left[G^{\leq 0}\right]$ and $k\left[H^{\leq 0}\right]$ follows from here by Lemma 2.9. Indeed, since $H$ is divisible, $H^{<0}$ does not have a greatest element. Hence any $f \in k\left[H^{\leq 0}\right]$ that is prime in $k\left[H^{\leq 0}\right]$ or in $k\left[G^{\leq 0}\right]$ must have nonzero constant term. Then by Lemma $2.9, f$ is prime in $k\left[H^{\leq 0}\right]$, resp. $k\left[G^{\leq 0}\right]$, iff $f$ is prime in $k[H]$, resp. $k[G]$.

From now on, we assume that $G$ is divisible, that is, a $\mathbb{Q}$-vector space.

Proposition 3.5 Let $G$ be a divisible ordered abelian group, $k$ a real closed field. If $f \in k\left[G^{\leq 0}\right]$ is a non-constant prime, then support $f$ does not lie in a 1-dimensional $\mathbb{Q}$-subspace of $G$.

Proof: Let support $f=\left\{g_{1}, \ldots, g_{n}, 0\right\}$. Write $f=a_{1} t^{g_{1}}+\cdots+a_{n} t^{g_{n}}+a_{0}$. Assume that $g_{i}=\frac{m_{i}}{n_{i}} g_{1}$ for $m_{i}, n_{i} \in \mathbb{N}$. Set $N=\operatorname{lcm}\left\{n_{i}\right\}, g=\frac{1}{3 N} g_{1}$, and $x=t^{g}$. Rewriting $f$ in terms of $x$, we obtain the polynomial $f(x)=a_{1} x^{N_{1}}+\cdots+a_{n} x^{N_{n}}+a_{0}$ (where $N_{i}=3 m_{i} M_{i}$ and $\left.N=M_{i} n_{i}\right)$. Since $f(x) \in k[x]$ has degree $\geq 3$, it factors in $k[x]$, which provides a factorization of $f$ in $k\left[G^{\leq 0}\right]$. 
Remark 3.6 In $[\mathrm{B} ;$ p.555] it is wrongly asserted that the support must be a $\mathbb{Q}$ linearly independent set. A counterexample is constructed as follows. Let $g_{1}, g_{2} \in$ $G^{<0}$ be $\mathbb{Q}$-linearly independent, then the element $f=t^{2 g_{1}}+t^{g_{1}+g_{2}}+t^{g_{1}}+t^{g_{2}}+1$ is prime in $k[G]$ by an argument similar to the proof of Proposition 3.8, below. Indeed, fix any $m \in \mathbb{N}$ and set set $x=t^{\frac{1}{m} g_{1}}, y=t^{\frac{1}{m} g_{2}}$, then rewriting $f$ in terms of $x$ and $y$, we obtain an irreducible polynomial $f(x, y)=x^{2 m}+\left(y^{m}+1\right) x^{m}+\left(y^{m}+1\right)$. This implies that $f$ is prime in $k[G]$ and, consequently, in $k\left[G^{\leq 0}\right]$.

Remark 3.7 Shepherdson's model mentioned at the beginning of the Introduction is the canonical integer part of $\mathbb{Q}\left(t^{g} \mid g \in \mathbb{Q}\right)^{r}$. By Proposition 2.4, this is just $\mathbb{Q}^{r}\left[\mathbb{Q}^{<0}\right] \oplus \mathbb{Z}$. By Proposition 3.5, a non-constant element cannot be prime. This explains why Shepherdson's model has no non-standard primes.

Next, we want to construct prime elements in $k\left[G^{\leq 0}\right]$. We first establish the following result:

Proposition 3.8 Let $G$ be a divisible torsion-free abelian group (resp., divisible ordered abelian group), $k$ a field of characteristic zero. Let $f$ be an element of $k[G]$ (resp., $\left.k\left[G^{\leq 0}\right]\right)$. Assume that support $f=\left\{g_{1}, \ldots, g_{n}, 0\right\}$ where $g_{1}, \ldots, g_{n}$ are linearly independent over $\mathbb{Q}$ and $n \geq 2$. Then $f$ is prime in $k[G]$ (resp., in $k\left[G^{\leq 0}\right]$ ).

Proof: Write $f=a_{1} t^{g_{1}}+\cdots+a_{n} t^{g_{n}}+a_{0}$ where $0 \neq a_{i} \in k$. Let $H=\left\langle g_{1}, \ldots, g_{n}\right\rangle_{\mathbb{Q}}$. First we show that $f$ is prime in $k[H]$. For $m \in \mathbb{N}$, let $H_{m}=\left\langle\frac{1}{m} g_{1}, \ldots, \frac{1}{m} g_{n}\right\rangle_{\mathbb{Z}}$. Then $k[H]$ is the direct limit of $k\left[H_{m}\right]$. By Lemma 2.8, it suffices to show that $f$ is prime in $k\left[H_{m}\right]$ for sufficiently large $m$.

Clearly, $k\left[H_{m}\right] \cong k\left[x_{1}^{ \pm 1}, \ldots, x_{n}^{ \pm 1}\right]$ where $x_{i}=t^{\frac{1}{m} g_{i}}$. Under this isomorphism $f$ corresponds to the polynomial $f_{m}\left(x_{1}, \ldots, x_{n}\right)=a_{1} x_{1}^{m}+\cdots+a_{n} x_{n}^{m}+1$. This polynomial is irreducible in $k\left[x_{1}, \ldots, x_{n}\right]$. One can show this e.g. by induction on $n \geq 2$ applying Eisenstein's criterion to $f_{m}\left(x_{1}, \ldots, x_{n}\right)=a_{1} x_{1}^{m}+\left(a_{2} x_{2}^{m}+\cdots+\right.$ $\left.a_{n} x_{n}^{m}+1\right)$ viewed as an element of $R\left[x_{1}\right]$ where $R=\bar{k}\left[x_{2}, \ldots, x_{n}\right]$ and $\bar{k}$ is the algebraic closure of $k$. For $n=2$, take $p=x_{2}-\xi \in R$ where $\xi$ is an $m$-th root of $-a_{2}^{-1}$, then $p$ satisfies the conditions of Eisenstein's criterion for $f_{m}$. For $n>2$, take $p=a_{2} x_{2}^{m}+\cdots+a_{n} x_{n}^{m}+1$, which is prime in $R$ by induction hypothesis and satisfies the conditions of Eisenstein's criterion for $f_{m}$.

Now by Lemma $2.9,1$ ) (with $R:=k$ and $T$ is generated by $x_{1}, \ldots, x_{n}$ ), $f_{m}$ is prime in $k\left[x_{1}^{ \pm 1}, \cdots, x_{n}^{ \pm 1}\right]$, so $f$ is prime in $k\left[H_{m}\right]$ for any $m$. Thus we have proved that $f$ is prime in $k[H]$. By Lemma 3.4, we conclude that $f$ is prime in $k[G]$. If $f \in k\left[G^{\leq 0}\right]$, then by Lemma $2.9,2), f$ is also prime in $k\left[G^{\leq 0}\right]$.

Example 3.9 Let $G$ be a divisible ordered abelian group, $k$ a real closed field. Consider $f=a_{1} t^{g_{1}}+a_{2} t^{g_{2}}+1$ where $g_{1}, g_{2} \in G^{<0}, 0 \neq a_{1}, a_{2} \in k$. Then $f$ is prime in $k\left[G^{\leq 0}\right]$ iff $g_{1}$ and $g_{2}$ are linearly independent over $\mathbb{Q}$.

Proof: Follows from Propositions 3.5 and 3.8. 
Corollary 3.10 Let $G$ be a divisible ordered abelian group, $k$ an ordered field. If $\operatorname{dim}_{\mathbb{Q}} G \geq 2$, then the ordered ring $k\left[G^{<0}\right] \oplus \mathbb{Z}$ has a cofinal set of primes.

Proof: $\quad$ Let $s \in k\left[G^{<0}\right] \oplus \mathbb{Z}$ and let $g=\min (\operatorname{support} s) \in G^{\leq 0}$. Then we can find $\mathbb{Q}$-linearly independent $g_{1}, g_{2} \in G^{<0}$ such that $g_{1}<g$. Let $f=t^{g_{1}}+t^{g_{2}}+1$. Then $f>s$ and $f$ is prime by Proposition 3.8.

\section{Primes and Irreducibles in $k\left(\left(G^{\leq 0}\right)\right)$.}

Throughout this section, $G$ is a divisible ordered abelian group, with rank $\Gamma, k$ a field of characteristic zero, and $F$ is a truncation closed subfield of $k((G))$ that contains $k(G)$. Note that it follows that the restriction of any series in $F$ to any convex subset of $G$ also belongs to $F$ (because the intersection of the support of the series with the convex subset can be written as the difference of two initial segments of the series). We want to investigate when a prime element of $k\left[G^{\leq 0}\right]$ remains prime or at least irreducible through the ring extensions

$$
k\left[G^{\leq 0}\right] \subset \operatorname{Neg}(F) \oplus k \subset k\left(\left(G^{\leq 0}\right)\right) .
$$

First we consider the following example to see how things can go wrong.

Example 4.1 Suppose we have $g_{1}, g_{2} \in G^{<0}$ such that $v\left(g_{1}\right)<v\left(g_{2}\right)$. Then $g_{1}$ and $g_{2}$ are linearly independent over $\mathbb{Q}$, so by Proposition 3.8, $f=t^{g_{1}}+t^{g_{2}}+1$ is prime in $k\left[G^{\leq 0}\right]$. However, $f$ can be factored in $k\left(\left(G^{\leq 0}\right)\right)$ as follows: $f=\left(t^{g_{2}}+1\right)\left(\frac{t^{g_{1}}}{t^{g_{2}}+1}+1\right)=$ $\left(t^{g_{2}}+1\right)\left(t^{g_{1}-g_{2}}\left(1+t^{-g_{2}}\right)^{-1}+1\right)=\left(t^{g_{2}}+1\right)\left(\sum_{n=1}^{\infty}(-1)^{n-1} t^{g_{1}-n g_{2}}+1\right)$. Thus $f$ does not remain irreducible in $k\left(\left(G^{\leq 0}\right)\right)$.

Generalizing this trick, we can show that if $f$ (non-constant) is irreducible in $\operatorname{Neg} F \oplus k$, then all nonzero elements of support $f$ must be archimedean equivalent, that is,

$$
\Gamma_{f}:=v(\operatorname{support} f \backslash\{0\})
$$

must be a singleton (this observation is due to Gonshor [G]). Indeed, let $\gamma=\min \Gamma_{f}$ and let $f_{\gamma}=\sum_{g: v(g)=\gamma} f_{g} t^{g}$. Since $F$ is truncation closed, $f_{\gamma} \in \operatorname{Neg}(F)$. Let $\varepsilon=f-f_{\gamma}$. If $\Gamma_{f}$ is not a singleton, then $\varepsilon \notin k$. Clearly, for all $g \in \operatorname{support} \varepsilon$, $v(g)>\gamma$. It follows that $f=\varepsilon\left(\frac{f_{\gamma}}{\varepsilon}+1\right)$ is a nontrivial factorization of $f$ in $\operatorname{Neg}(F) \oplus k$.

This phenomenon suggests that the problems of irreducibility and primality in $\operatorname{Neg}(F) \oplus k$ should essentially reduce to the case when $G$ is archimedean. Let $f$ be an element of $\operatorname{Neg}(F) \oplus k$ such that $\Gamma_{f}=\{\gamma\}$. Fix a complement for $D_{\gamma}$ in $C_{\gamma}$ so that we can view the archimedean component $A_{\gamma}$ as a subgroup of $C_{\gamma}$. Then $C_{\gamma}=A_{\gamma} \oplus D_{\gamma}$ (lexicographic sum). Consequently, $k\left(\left(C_{\gamma}\right)\right)=k\left(\left(D_{\gamma}\right)\right)\left(\left(A_{\gamma}\right)\right)$ and

$$
k\left(\left(C_{\gamma}^{<0}\right)\right)=k\left(\left(D_{\gamma}\right)\right)\left(\left(A_{\gamma}^{<0}\right)\right) \oplus k\left(\left(D_{\gamma}^{<0}\right)\right) .
$$


Set $k_{\gamma}=k\left(\left(D_{\gamma}\right)\right) \cap F$ and $F_{\gamma}=k\left(\left(C_{\gamma}\right)\right) \cap F$. Then $f \in F_{\gamma}$. Also from truncation closedness of $F$ it follows that $F_{\gamma}=k_{\gamma}\left(\left(A_{\gamma}\right)\right) \cap F$, since each coefficient that appears when we represent a series from $F_{\gamma}$ as an element of $k\left(\left(D_{\gamma}\right)\right)\left(\left(A_{\gamma}\right)\right)$, is the restriction of the series to a convex subset of $C_{\gamma}$, divided by a monomial, and thus also belongs to $F$. So $F_{\gamma}$ is a truncation closed subfield of $k_{\gamma}\left(\left(A_{\gamma}\right)\right)$ containing $k_{\gamma}\left(A_{\gamma}\right)$. Viewing the elements of $k_{\gamma}$ as constants, define $\operatorname{Neg}_{k_{\gamma}}\left(F_{\gamma}\right)=F_{\gamma} \cap k_{\gamma}\left(\left(A_{\gamma}^{<0}\right)\right)$. Since we are changing the field of constants, we have to indicate by a subscript over which ground field each Neg is taken. In particular, what was previously denoted by $\operatorname{Neg}(F)$ now will be written as $\operatorname{Neg}_{k}(F)$.

Theorem 4.2 Let $f$ be an element of $\operatorname{Neg}_{k}(F) \oplus k$ with constant term $f_{0} \in k$. Assume that $\Gamma_{f}=\{\gamma\}$. Then $f$ is prime (resp., irreducible) in $\mathrm{Neg}_{k}(F) \oplus k$ iff the following two conditions hold: 1) $f$ is prime (resp., irreducible) in $\mathrm{Neg}_{k_{\gamma}}\left(F_{\gamma}\right) \oplus k_{\gamma}$ and 2) either $f_{0} \neq 0$ or $\gamma=\max \Gamma$.

Proof: First we observe that if condition 2) does not hold, that is, $f_{0}=0$ and there exists $g_{0} \in G^{<0}$ such that $v\left(g_{0}\right)>\gamma$, then $f$ can be factored in $\operatorname{Neg}_{k}(F) \oplus k$ : $f=t^{g_{0}}\left(f t^{-g_{0}}\right)$. So from now on we assume that condition 2) holds. The proof that $f$ is prime (resp., irreducible) in $\operatorname{Neg}_{k}(F) \oplus k$ iff $f$ is prime (resp., irreducible) in $\operatorname{Neg}_{k_{\gamma}}\left(F_{\gamma}\right) \oplus k_{\gamma}$ will proceed in two steps.

Step 1. We claim that $f$ is prime (resp., irreducible) in $\operatorname{Neg}_{k_{\gamma}}\left(F_{\gamma}\right) \oplus k_{\gamma}$ iff $f$ is prime (resp., irreducible) in $\operatorname{Neg}_{k}\left(F_{\gamma}\right) \oplus k$.

If $\gamma=\max \Gamma$, then $k_{\gamma}=k$ and the claim is trivial. So assume $f_{0} \neq 0$. Replacing $f$ by $f / f_{0}$, we can assume that $f$ has constant term 1 as an element of $k((G))$. Since $\Gamma_{f}=\{\gamma\}, f$ still has constant term 1 when viewed as an element of $k_{\gamma}\left(\left(A_{\gamma}\right)\right)$. By Lemma 2.7 with $G:=A_{\gamma}, K:=k_{\gamma}, \operatorname{Neg}:=\operatorname{Neg}_{k_{\gamma}}\left(F_{\gamma}\right)$, and $R:=\operatorname{Neg}_{k}\left(k_{\gamma}\right) \oplus k$, $f$ is prime (resp., irreducible) in Neg $\oplus K=\operatorname{Neg}_{k_{\gamma}}\left(F_{\gamma}\right) \oplus k_{\gamma}$ iff $f$ is prime (resp., irreducible) in Neg $\oplus R=\operatorname{Neg}_{k_{\gamma}}\left(F_{\gamma}\right) \oplus \operatorname{Neg}_{k}\left(k_{\gamma}\right) \oplus k$. It remains to observe that decomposition (3) implies that $\operatorname{Neg}_{k}\left(F_{\gamma}\right)=\operatorname{Neg}_{k_{\gamma}}\left(F_{\gamma}\right) \oplus \operatorname{Neg}_{k}\left(k_{\gamma}\right)$.

Step 2. We claim that $f$ is prime (resp., irreducible) in $\operatorname{Neg}_{k}\left(F_{\gamma}\right) \oplus k$ iff $f$ is prime (resp., irreducible) in $\operatorname{Neg}_{k}(F) \oplus k$.

Let $E_{\gamma}$ be a complement for $C_{\gamma}$ in $G$. Then $G=E_{\gamma} \oplus C_{\gamma}$ (lexicographic sum). Consequently, $k((G))=k\left(\left(C_{\gamma}\right)\right)\left(\left(E_{\gamma}\right)\right)$ and

$$
k\left(\left(G^{<0}\right)\right)=k\left(\left(C_{\gamma}\right)\right)\left(\left(E_{\gamma}^{<0}\right)\right) \oplus k\left(\left(C_{\gamma}^{<0}\right)\right) .
$$

Since $F$ is truncation closed, $F \subset F_{\gamma}\left(\left(E_{\gamma}\right)\right)$. It remains to apply Lemma 2.6 with $G:=E_{\gamma}, K:=F_{\gamma}, \operatorname{Neg}:=\operatorname{Neg}_{F_{\gamma}}(F)$, and $R:=\operatorname{Neg}_{k}\left(F_{\gamma}\right) \oplus k$ and observe that Neg $\oplus R=\operatorname{Neg}_{k}(F) \oplus k$ since $\operatorname{Neg}_{k}(F)=\operatorname{Neg}_{F_{\gamma}}(F) \oplus \operatorname{Neg}_{k}\left(F_{\gamma}\right)$ by (4).

Taking $F=k((G))$, we obtain the following important

Corollary 4.3 Let $f$ be an element of $k\left(\left(G^{\leq 0}\right)\right)$ with constant term $f_{0}$. Assume that $\Gamma_{f}=\{\gamma\}$. Then $f$ is prime (resp., irreducible) in $k\left(\left(G^{\leq 0}\right)\right)$ iff the following two conditions hold: 1) $f$ is prime (resp., irreducible) in $k_{\gamma}\left(\left(A_{\gamma}^{\leq 0}\right)\right)$ where $k_{\gamma}=k\left(\left(D_{\gamma}\right)\right)$ and 2) either $f_{0} \neq 0$ or $\gamma=\max \Gamma$. 
As an application of Corollary 4.3, we prove the following generalizations of [B; Theorem 11.2]. First we introduce some notation. Let $f$ be an arbitrary element of $\operatorname{Neg}(F) \oplus k$ and let $\gamma \in \Gamma_{f}$. As before, set $f_{\gamma}=\sum_{g: v(g)=\gamma} f_{g} t^{g}$. Then $f_{\gamma} \in F_{\gamma}=$ $k\left(\left(C_{\gamma}\right)\right) \cap F$. Set

$$
S_{f}(\gamma):=\pi_{\gamma}\left(\operatorname{support} f_{\gamma}\right) \subset A_{\gamma} .
$$

Once a complement for $D_{\gamma}$ in $C_{\gamma}$ is fixed, we can view $F_{\gamma}$ as a subfield of $k_{\gamma}\left(\left(A_{\gamma}\right)\right)$, where $k_{\gamma}=k\left(\left(D_{\gamma}\right)\right) \cap F$ as before. Then $S_{f}(\gamma)$ is just the support of $f_{\gamma}$ viewed as an element of $k_{\gamma}\left(\left(A_{\gamma}\right)\right)$.

Corollary 4.4 Assume that $\Gamma_{f}=\{\gamma\}, S_{f}(\gamma)$ is finite, and $f$ is prime as an element of $k_{\gamma}\left[A_{\gamma}^{\leq 0}\right]$. Then $f$ is irreducible in $k\left(\left(G^{\leq 0}\right)\right)$.

Proof: $\quad$ Since $A_{\gamma}$ is archimedean, $f$ remains irreducible in $k_{\gamma}\left(\left(A_{\gamma}^{\leq 0}\right)\right)$ by Berarducci's result. Now apply Corollary 4.3.

Corollary 4.5 Let $f$ be prime in $k\left[G^{\leq 0}\right]$ and suppose that support $f$ generates an archimedean subgroup of $G$. Then $f$ remains irreducible in $k\left(\left(G^{\leq 0}\right)\right)$ and, consequently, in $\operatorname{Neg}(F) \oplus k$, for any truncation closed $F, k(G) \subset F \subset k((G))$.

Proof: Let $H$ be the subgroup generated by support $f$. Since $H$ is archimedean, all nonzero elements of $H$ have the same valuation, say $\gamma$. Moreover, we can pick a complement $A_{\gamma}$ for $D_{\gamma}$ in $C_{\gamma}$ in such a way that $H \subset A_{\gamma}$. Since $f$ is prime in $k\left[G^{\leq 0}\right]$, the constant term $f_{0} \neq 0$. By Corollary 4.4, it suffices to show that $f$ is prime in $k_{\gamma}\left[A_{\gamma}^{\leq 0}\right]$. By the choice of $A_{\gamma}, f \in k\left[A_{\gamma}^{\leq 0}\right]$. By Lemma 3.4, $f$ is prime in $k\left[A_{\gamma}^{\leq 0}\right]$, so $R:=k\left[A_{\gamma}^{\leq 0}\right] /(f)$ is a domain. It follows that $R\left(\left(D_{\gamma}\right)\right)$ is also a domain. But $R\left(\left(D_{\gamma}\right)\right)=\left(k\left[A_{\gamma}^{\leq 0}\right] /(f)\right)\left(\left(D_{\gamma}\right)\right)=k\left[A_{\gamma}^{\leq 0}\right]\left(\left(D_{\gamma}\right)\right) /(f)=k\left(\left(D_{\gamma}\right)\right)\left[A_{\gamma}^{\leq 0}\right] /(f)=$ $k_{\gamma}\left[A_{\gamma}^{\leq 0}\right] /(f)$. We conclude that $f$ is prime in $k_{\gamma}\left[A_{\gamma}^{\leq 0}\right]$.

Example 4.6 Consider $f=a_{1} t^{g_{1}}+a_{2} t^{g_{2}}+1$ where $g_{1}, g_{2} \in G^{<0}$ are $\mathbb{Q}$-linearly independent and $0 \neq a_{1}, a_{2} \in k$. As we know, $f$ is prime in $k\left[G^{\leq 0}\right]$. It remains irreducible in $k\left(\left(G^{\leq 0}\right)\right)$ iff $v\left(g_{1}\right)=v\left(g_{2}\right)$ and $g_{1}, g_{2}$ are $\mathbb{Q}$-valuation independent.

Proof: If the conditions on $g_{1}, g_{2}$ are satisfied, then the subgroup $\left\langle g_{1}, g_{2}\right\rangle$ is archimedean by Corollary 1.3, so $f$ remains irreducible in $k\left(\left(G^{\leq 0}\right)\right)$ by Corollary 4.5. Now if $v\left(g_{1}\right) \neq v\left(g_{2}\right)$, then, as we already saw in Example 4.1, $f$ does not remain irreducible in $k\left(\left(G^{\leq 0}\right)\right)$. If $v\left(g_{1}\right)=v\left(g_{2}\right)=\gamma$, but $g_{1}, g_{2}$ are not $\mathbb{Q}$-valuation independent, then there exist $m, n \in \mathbb{N}$ such that $v(g)>\gamma$ where $g=n g_{1}-m g_{2}$. In this case, let $x=t^{\frac{1}{3 m} g_{1}}$, then $f=x^{3 m}+\alpha x^{3 n}+1$ where $\alpha=t^{-\frac{1}{m} g} \in k_{\gamma}=k\left(\left(D_{\gamma}\right)\right)$. Clearly, if $k$ is real closed, this polynomial factors in $k_{\gamma}[x]$, so $f$ does not remain irreducible in $k\left(\left(G^{\leq 0}\right)\right)$.

This example shows that in Corollary 4.5 the condition that support $f$ generates an archimedean subgroup cannot be weakened to say just that $\Gamma_{f}$ is a singleton. On the other hand, consider $f^{\prime}=t^{2 g_{1}}+t^{g_{1}+g_{2}}+t^{g_{1}-g}+t^{g_{2}+g}+1$ where $g_{1}, g_{2} \in G^{<0}$ 
are $\mathbb{Q}$-valuation independent and $v\left(g_{1}\right)=v\left(g_{2}\right)=\gamma<v(g)$. If $g \neq 0$, then the subgroup generated by support $f^{\prime}$ is not archimedean, but $f^{\prime}$ is still irreducible in $k\left(\left(G^{\leq 0}\right)\right)$. Indeed, choose $A_{\gamma}$ such that $g_{1}, g_{2} \in A_{\gamma}$. Then $f^{\prime}$ is prime in $k_{\gamma}\left[A_{\gamma}^{\leq 0}\right]$ : $f^{\prime}=t^{2 g_{1}}+\alpha^{-1}\left(\alpha t^{g_{2}}+1\right) t^{g_{1}}+\left(\alpha t^{g_{2}}+1\right)$ where $\alpha=t^{g} \in k_{\gamma}$, so by rewriting $f^{\prime}$ in terms of $x:=t^{\frac{1}{m} g_{1}}$ and $y:=t^{\frac{1}{m} g_{2}}(m \in \mathbb{N})$, we obtain an irreducible polynomial $x^{2 m}+\alpha^{-1}\left(\alpha y^{m}+1\right) x^{m}+\left(\alpha y^{m}+1\right)$ (cf. Remark 3.6). Therefore, by Corollary 4.4, $f^{\prime}$ is irreducible in $k\left(\left(G^{\leq 0}\right)\right)$.

We do not know in general in Corollary 4.5 if $f$ actually remains prime in $\operatorname{Neg}(F) \oplus$ $k$, but we can show this in the case when the field $F$ is "small".

Theorem 4.7 Assume that for any $f \in \operatorname{Neg}(F) \oplus k$ and any $\gamma \in \Gamma_{f}$, the set $S_{f}(\gamma)$ is finite. Then every irreducible element of $\operatorname{Neg}(F) \oplus k$ is prime.

Proof: Let $f$ be irreducible in $\operatorname{Neg}(F) \oplus k$. Then $\Gamma_{f}=\{\gamma\}$ for some $\gamma \in \Gamma$. By Theorem 4.2, $f$ is irreducible in $\operatorname{Neg}_{k_{\gamma}}\left(F_{\gamma}\right) \oplus k_{\gamma}$. By our assumption on $F$, $\operatorname{Neg}_{k_{\gamma}}\left(F_{\gamma}\right) \oplus k_{\gamma}=k_{\gamma}\left[A_{\gamma}^{\leq 0}\right]$. By Corollary 3.2, $f$ is prime in $k_{\gamma}\left[A_{\gamma}^{\leq 0}\right]$. Hence by Theorem 4.2, $f$ is prime in $\operatorname{Neg}(F) \oplus k$.

One important case when the sets $S_{f}(\gamma)$ are finite is $F=k(G)^{r}$, the field of algebraic power series. In the following corollaries we assume that $k$ is a real closed field.

Corollary 4.8 Let $F=k(G)^{r}$. Then every irreducible element of $\operatorname{Neg}(F) \oplus k$ is prime.

Proof: By Theorem 4.7, it suffices to show that for any $f \in k\left(\left(G^{\leq 0}\right)\right)$ which is algebraic over $k(G)$ and any $\gamma \in \Gamma_{f}$, the set $S_{f}(\gamma)$ is finite. (As a matter of fact, the set $\Gamma_{f}$ is also finite, but we do not need this property here.) By truncation closedness of $F$, we can assume without loss of generality that $f=f_{\gamma}$.

We claim that $f$ is algebraic over $k\left(C_{\gamma}\right)$. Indeed, since $f$ is algebraic over $k(G)$, there exists a nonzero polynomial $p(x) \in k[G][x]$ such that $p(f)=0$. Choose a complement $E_{\gamma}$ for $C_{\gamma}$ in $G$. Then $k[G]=k\left[C_{\gamma}\right]\left[E_{\gamma}\right]$ and so we can write $p(x)=$ $\sum_{g \in E_{\gamma}} p_{g}(x) t^{g}$ (finite sum) where $p_{g}(x) \in k\left[C_{\gamma}\right][x]$. Since $p(x)$ is nonzero, there exists $g_{0} \in E_{\gamma}$ such that $p_{g_{0}}(x)$ is nonzero. Since $p(f)=0$ and the set $\left\{t^{g} \mid g \in E_{\gamma}\right\}$ is linearly independent over $k\left(\left(C_{\gamma}\right)\right)$, it follows that $p_{g_{0}}(f)=0$, which proves the claim.

Now let $K=k\left(\left(D_{\gamma}\right)\right)$. Then $f \in K\left(\left(A_{\gamma}^{\leq 0}\right)\right)$. Since $k\left(C_{\gamma}\right) \subset K\left(A_{\gamma}\right), f$ is algebraic over $K\left(A_{\gamma}\right)$. But $A_{\gamma}$ is archimedean, so by Proposition 2.4, the support of $f$, viewed as an element of $K\left(\left(A_{\gamma}\right)\right)$, is finite. As noted earlier, this support is precisely $S_{f}(\gamma)$.

Example 4.9 Let $F=k(G)^{r}$. Let $g_{1}, g_{2} \in G^{<0}$ be $\mathbb{Q}$-valuation independent and $v\left(g_{1}\right)=v\left(g_{2}\right)$. Consider $f=a_{1} t^{g_{1}}+a_{2} t^{g_{2}}+1$ where $0 \neq a_{1}, a_{2} \in k$. Then $f$ is prime in $\operatorname{Neg}(F) \oplus k$. 
Proof: Combine Corollaries 4.5 and 4.8 .

Corollary 4.10 Assume that for every $\gamma \in \Gamma$ we have $\operatorname{dim}_{\mathbb{Q}} A_{\gamma} \geq 2$. Then for any truncation closed $F, k(G) \subset F \subset k((G))$, the ordered ring $\operatorname{Neg}(F) \oplus \mathbb{Z}$ has a cofinal set of irreducible elements with finite support.

Proof: $\quad$ Let $s \in \operatorname{Neg}(F) \oplus \mathbb{Z}$ and let $g=\min ($ support $s) \in G^{\leq 0}$. Take $g_{1}<g$ and set $\gamma=v\left(g_{1}\right)$. Choose $A_{\gamma}$ such that $g_{1} \in A_{\gamma}$. By hypothesis, we can find $g_{2} \in A_{\gamma}$ linearly independent from $g_{1}$. Without loss of generality, $g_{2}<0$. Then $f=t^{g_{1}}+t^{g_{2}}+1$ is irreducible in $\operatorname{Neg}(F) \oplus \mathbb{Z}$ by Corollary 4.5. Clearly, $f>s$.

Corollary 4.11 Assume that for every $\gamma \in \Gamma$ we have $\operatorname{dim}_{\mathbb{Q}} A_{\gamma} \geq 2$. Let $F=$ $k(G)^{r}$. Then the ordered ring $\operatorname{Neg}(F) \oplus \mathbb{Z}$ has a cofinal set of primes with finite support.

Proof: $\quad$ Combine Corollaries 4.10 and 4.8 .

As another application of Corollary 4.3, we obtain the following improvement of [P; Theorem 4.2].

Theorem 4.12 Let $s \in k\left(\left(G^{<0}\right)\right)$ be such that all elements of support s have the same valuation $\gamma \in \Gamma$. Assume also that the order type of the set $\pi_{\gamma}($ support $s) \subset$ $A_{\gamma}^{<0}$ is $\omega$ and the least upper bound is 0 . Then $s+1$ is prime in $k\left(\left(G^{\leq 0}\right)\right)$.

Proof: By Corollary 4.3, it suffices to show that $f:=s+1$ is prime in $k_{\gamma}\left(\left(A_{\gamma}^{\leq 0}\right)\right)$ where $k_{\gamma}=k\left(\left(D_{\gamma}\right)\right)$. Since $A_{\gamma}$ is archimedean and the set $S_{f}(\gamma)=\pi_{\gamma}($ support $s)$ is precisely the support of $s$ viewed as an element of $k_{\gamma}\left(\left(A_{\gamma}\right)\right)$, we can apply Pitteloud's result to conclude that $f$ is indeed prime in $k_{\gamma}\left(\left(A_{\gamma}^{\leq 0}\right)\right)$.

Corollary 4.13 Let $s \in k\left(\left(G^{<0}\right)\right)$ be such that the support of $s$ is of order type $\omega$ and is contained in an archimedean subgroup $A$ of $G$. Assume that $s$ is not divisible by any monomial $t^{a}$ with $a \in A^{<0}$. Then $s+1$ is prime in $k\left(\left(G^{\leq 0}\right)\right)$.

Example 4.14 For any $g \in G^{<0}$, the series

$$
p_{g}:=\sum_{n=1}^{\infty} t^{\frac{1}{n} g}+1
$$

is prime in $k\left(\left(G^{\leq 0}\right)\right)$.

Example 4.15 Let $g, g^{\prime} \in G^{<0}$ be such that $v\left(g^{\prime}\right)>v(g)$. Then the series

$$
p_{g, g^{\prime}}:=\left(\sum_{n=1}^{\infty} \sum_{m=1}^{\infty} t^{\frac{1}{n} g+\frac{1}{m} g^{\prime}}\right)+1
$$

is prime in $k\left(\left(G^{\leq 0}\right)\right)$. Note that the support of $f:=p_{g, g^{\prime}}-1$ has order type $\omega^{2}$, but $S_{f}(\gamma)$, where $\gamma=v(g)$, has order type $\omega$, so Theorem 4.12 applies. 
Corollary 4.16 The ordered ring $k\left(\left(G^{<0}\right)\right) \oplus \mathbb{Z}$ has a cofinal set of prime elements with infinite support.

Proof: $\quad$ Let $s \in k\left(\left(G^{<0}\right)\right) \oplus \mathbb{Z}$ and let $g=\min ($ support $s) \in G^{\leq 0}$. Pick $g^{\prime}<g$, then $p_{g^{\prime}}$ is prime by Example 4.14 and $p_{g^{\prime}}>s$.

\section{Exponential Integer Parts of Exponential Fields}

In this Section, we apply the results of the previous sections to study EIP's of exponential fields. Let $(K,+, \cdot, 0,1,<)$ be a real closed field $(\mathrm{RCF})$. We say that $K$ is an exponential field if there exists an exponential on $K$, that is, a map exp such that

(EXP) $\quad \exp :(K,+, 0,<) \simeq\left(K^{>0}, \cdot, 1,<\right)$ (that is, exp is an isomorphism of ordered groups).

We shall further consider exponentials that satisfy the growth axiom scheme:

$$
\text { for all } x \in K, x \geq n^{2} \Longrightarrow \exp (x)>x^{n} \quad(n \geq 1),
$$

Thus $(K, \exp )$ is a model for the fragment

$$
T_{E X P}=R C F+E X P+G A
$$

of $\operatorname{Th}(\mathbb{R}, \exp ):=$ the elementary theory of the ordered field of the reals with exponentiation.

A logarithm on $K$ is the compositional inverse $\log =\exp ^{-1}$ of an exponential.

As before, we consider the residue field $k$ embedded into $K$. By appropriately modifying exp (see [K; Lemma 1.18]), we can always assume that

$$
\exp \left(\mathcal{O}_{K}\right)=\mathcal{U}_{K}^{>0} \quad \text { and } \quad \exp \left(\mathcal{M}_{K}\right)=1+\mathcal{M}_{K} \quad \text { and } \quad \exp (k)=k^{>0} .
$$

Theorem 5.1 Every truncation integer part of an exponential field has a cofinal set of irreducibles.

Proof: We can apply Corollary 4.10 because the condition on $G$ is always fulfilled when $G$ is the value group of an exponential field (see [K; Proposition 1.22]).

An exponential integer part (EIP) of $K$ is an integer part $Z$ such that for any $z \in Z^{>0}$, we have $2^{z} \in Z^{>0}$ (where by definition $2^{x}:=\exp (x \log 2$ ) for all $x \in K$ ). The following easy proposition tells us when a truncation IP is actually an EIP.

Proposition 5.2 Let $F$ be a truncation closed subfield of $k((G))$ that contains $k(G)$. Assume that $F$ admits an exponential exp such that $\exp (\operatorname{Neg}(F)) \subset \operatorname{Mon}(F)$ where $\operatorname{Mon}(F):=\operatorname{Mon} k((G))$. Then the canonical integer part $Z_{F}=\operatorname{Neg}(F) \oplus \mathbb{Z}$ is an exponential integer part. 
Proof: $\quad$ Let $z=a+z_{0} \in Z_{F}^{>0}, a \in \operatorname{Neg}(F), z_{0} \in \mathbb{Z}$. If $a=0$ then $z_{0}>0$ and $2^{z_{0}} \in \mathbb{Z}$. If not, then $a>0$ and $2^{a+z_{0}}=2^{z_{0}} 2^{a}=2^{z_{0}} \exp (a \log 2)$. Since $\log 2 \in k$ and $\operatorname{Neg}(F)$ is a $k$-vector space, $a \log 2 \in \operatorname{Neg}(F)$. So $\exp (a \log 2)=t^{g}$ for some $g \in G$. Since $a \log 2>0, t^{g}>1$, so $g<0$. Therefore, $2^{a+z_{0}}=2^{z_{0}} t^{g} \in \operatorname{Neg}(F) \subset Z_{F}$ as required.

Theorem 5.3 Every exponential field has an exponential integer part with a cofinal set of irreducibles.

Proof: In $[\mathrm{R}]$ it is shown that given an exponential field $K$, there is a truncation closed embedding $\varphi$ of $K$ into $k((G))$ such that $\exp (\operatorname{Neg}(F)) \subset \operatorname{Mon}(F)$ where $F:=\varphi(K)$, so $\operatorname{Neg}(F) \oplus \mathbb{Z}$ is an EIP of $F$. It follows that $K$ has a truncation IP which is an EIP, thus the assertion follows by Theorem 5.1.

In the following example, we describe a procedure to construct countable nonarchimedean models of $T_{E X P}$ which are algebraic power series fields, and investigate their canonical IP's. We recall that a real closed field $K$ is an ordered vector space over the residue field $k$, and all archimedean components of $(K,+, 0,<)$ have dimension 1 over $k$.

\section{Example 5.4 : Countable Fields of Algebraic Power Series with Expo- nentiation.}

Let $E$ be a countable exponential subfield of ( $\mathbb{R}$, exp) (for example, take $E$ to be the smallest real closed, exp-closed and log-closed subfield of $\mathbb{R})$. Set $G=\oplus_{\mathbb{Q}} E$ the lexicographic sum over $\mathbb{Q}$ of copies of $(E,+, 0,<)$, that is,

$$
G:=\left\{\sum_{q \in \mathbb{Q}} g_{q} 1_{q} \mid g_{q} \in E, g_{q}=0 \text { for all but finitely many } q \in \mathbb{Q}\right\}
$$

endowed with pointwise addition and lexicographic order (here, $1_{q}$ denotes the characteristic function on the singleton $\{q\})$. Set $\mathbf{E}=E(G)^{r}$, then $\mathbf{E}$ is countable and real closed. Further $\mathbf{E}$ is a truncation closed subfield of $E((G))[\mathrm{M}-\mathrm{R}]$.

We want an exponential exp (or equivalently, a logarithm $\log$ ) on $\mathbf{E}$ such that (E, exp) is a model of $T_{E X P}$, and such that the canonical IP $Z_{\mathbf{E}}=\operatorname{Neg}(\mathbf{E}) \oplus \mathbb{Z}$ is an EIP.

Observe first that

$$
\begin{aligned}
(\mathbf{E},+, 0,<) & =\operatorname{Neg}(\mathbf{E}) \oplus \mathcal{O}_{\mathbf{E}} . \\
\left(\mathbf{E}^{>0}, \cdot, 1,<\right) & =\operatorname{Mon}(\mathbf{E}) \times \mathcal{U}_{\mathbf{E}}^{>0} .
\end{aligned}
$$

We shall define $\log$ on $\mathbf{E}^{>\mathbf{0}}$ as follows. We first define $\log$ on $\mathcal{U}_{\mathbf{E}}^{>0}=E^{>0} \times\left(1+\mathcal{M}_{\mathbf{E}}\right)$. By $\left[K\right.$; Theorem 2.22] there exists a $\mathbb{Q}$-valuation basis $B$ of $\mathcal{M}_{\mathbf{E}}$ such that the set $\{1+b \mid b \in B\}$ is a $\mathbb{Q}$-valuation basis of $1+\mathcal{M}_{\mathbf{E}}$. Define $\log (1+b):=b$ and extend $\log$ to $1+\mathcal{M}_{\mathbf{E}}$ by linearity. One proves that $\log$ is an isomorphism of ordered groups with $\log \left(1+\mathcal{M}_{\mathbf{E}}\right)=\mathcal{M}_{\mathbf{E}}\left[\mathrm{K}\right.$; Lemma 2.21]. For $u \in \mathcal{U}_{\mathbf{E}}^{>0}$, write $u=r y$ 
where $r \in E^{>0}, y \in 1+\mathcal{M}_{\mathbf{E}}$, and define $\log u:=\log r+\log y$ (where $\log r \in \mathbb{R}$ is the natural logarithm). Using these definitions, it is easily seen that log is an isomorphism of ordered groups with $\log \left(\mathcal{U}_{\mathbf{E}}^{>0}\right)=\mathcal{O}_{\mathbf{E}}$.

Now we define $\log$ on Mon $(\mathbf{E})$. By [K; Proposition 2.16], fix an isomorphism of chains $s: \mathbb{Q} \rightarrow G^{<0}$ such that for all $g \in G^{<0}$

$$
s(v(g))>g .
$$

Note that $\left\{1_{q} \mid q \in \mathbb{Q}\right\}$ is a strongly independent $E$-basis of $G$. Now $\operatorname{Neg}(\mathbf{E})$ is a countable ordered $E$-vector space with 1-dimensional archimedean components, so it admits a strongly independent $E$-basis.

Fix such a basis $\left\{b_{s(q)} \mid q \in \mathbb{Q}\right\}$ with $V\left(b_{s(q)}\right)=s(q)$ and $b_{s(q)}<0$ for all $q \in \mathbb{Q}$. Define $\log \left(t^{1_{q}}\right):=b_{s(q)}$ for all $q \in \mathbb{Q}$, and extend $\log$ to Mon $(\mathbf{E})$ by linearity:

$$
\log \left(t^{g}\right)=\sum_{q \in \mathbb{Q}} g_{q} b_{s(q)} \text { for } g=\sum_{q \in \mathbb{Q}} g_{q} 1_{q} .
$$

It is easily verified that $\log$ is an isomorphism of ordered groups with $\log (\operatorname{Mon}(\mathbf{E}))=$ $\operatorname{Neg}(\mathbf{E})$.

We extend $\log$ to $\mathbf{E}^{>0}$ using the above definitions via the decomposition (6). The inequality (7) implies that $\exp :=\log ^{-1}$ satisfies (GA) [K; Theorem 2.5]. Thus $(\mathbf{E}, \exp )$ is a model of $T_{E X P}$. By Proposition $5.2, Z_{\mathbf{E}}=\operatorname{Neg}(\mathbf{E}) \oplus \mathbb{Z}$ is an EIP. By Corollary 4.11, it has a cofinal set of primes (with finite support).

\section{Example 5.5 : Exponential-Logarithmic Power Series Fields.}

We describe the canonical integer part of the Exponential-Logarithmic Power Series Fields (EL-series fields for short). The EL-series fields are models of $T h(\mathbb{R}, \exp )$ constructed in [K; Chapter 5 p. 79].

The EL-series field $\mathbb{R}((\Gamma))^{E L}$ is a countable increasing union of power series fields with exponents in properly chosen value groups $G_{n}$ :

$$
\mathbb{R}((\Gamma))^{E L}=\bigcup_{n \in \mathbb{N}} \mathbb{R}\left(\left(G_{n}\right)\right) .
$$

The field $\mathbb{R}((\Gamma))^{E L}$ is a proper subfield of $\mathbb{R}\left(\left(G_{\omega}\right)\right)$ where

$$
G_{\omega}:=\bigcup_{n \in \mathbb{N}} G_{n}
$$

In fact, $\mathbb{R}((\Gamma))^{E L}$ is truncation closed and contains $\mathbb{R}\left(G_{\omega}\right)$ (so its value group is $\left.G_{\omega}\right)$. Furthermore,

$$
\operatorname{Neg}\left(\mathbb{R}((\Gamma))^{E L}\right)=\bigcup_{n \in \mathbb{N}} \operatorname{Neg} \mathbb{R}\left(\left(G_{n}\right)\right) .
$$

Therefore, the truncation integer part of $\mathbb{R}((\Gamma))^{E L}$ is

$$
Z_{E L}=\bigcup_{n \in \mathbb{N}} Z_{n}
$$


where $Z_{n}:=\mathbb{R}\left(\left(G_{n}^{<0}\right)\right) \oplus \mathbb{Z}$ is the canonical integer part of $\mathbb{R}\left(\left(G_{n}\right)\right)$. The construction of $\mathbb{R}((\Gamma))^{E L}$ guarantees that

$$
\exp \left(\operatorname{Neg}\left(\mathbb{R}((\Gamma))^{E L}\right)\right)=\operatorname{Mon}\left(\mathbb{R}((\Gamma))^{E L}\right) .
$$

By Proposition 5.2 we have that $Z_{E L}$ is indeed an EIP of $\mathbb{R}((\Gamma))^{E L}$. We now show that $Z_{E L}$ has a cofinal set of primes (with infinite support):

Proposition 5.6 The canonical exponential integer part $Z_{E L}$ of $\mathbb{R}((\Gamma))^{E L}$ has a cofinal set of primes with infinite support.

Proof: Let $g \in G_{\omega}^{<0}$. Then $g \in G_{n}$ for some $n \in \mathbb{N}$ and the series $p_{g}=\sum_{l=1}^{\infty} t^{\frac{g}{l}}+1 \in$ $\mathbb{R}\left(\left(G_{n}\right)\right)$ is prime in $Z_{m}$ for all $m \geq n$ by Example 4.14 . Therefore, by Lemma 2.8, $p_{g}$ remains prime in $Z_{E L}=\bigcup_{n \in \mathbb{N}} Z_{n}$. The set $\left\{p_{g} \mid g \in G_{\omega}^{<0}\right\}$ is clearly cofinal in $\mathbb{R}\left(\left(G_{\omega}\right)\right)$ and thus in $\mathbb{R}((\Gamma))^{E L}$.

\section{References}

[B] Berarducci, A.: Factorization in generalized power series, Trans. Amer. Math. Soc. 352 No. 2 (2000), 553-577

[Bi] Biljakovic, D. : Recursive Models of Open Induction With Infinite Primes, preprint (1996)

[Bo] Boughattas, S. : Résultats optimaux sur l'existence d'une Partie Entière dans les corps ordonnés, Journal of Symbolic Logic, 58 (1993), 326-333

[Bo2] Boughattas, S. : Trois théorèmes sur l'induction pour les formules ouvertes munies de l'exponentielle, Journal of Symbolic Logic, 65 (2000), $111-154$

[Br] Brown, R.: Valued vector spaces of countable dimension, Publ. Math. Debrecen 18 (1971), 149-151

[B-O] Berarducci, A. - Otero, M.: A Recursive Nonstandard Model of Normal Open Induction, Journal of Symbolic Logic, 61 (1996), 1228-1241

[F] Fornasiero, A.:Embedding Henselian fields into power series, preprint (2004) http://www.dm.unipi.it/ fornasiero/ressayre.pdf

[G] Gonshor, H.:An introduction to the theory of surreal numbers, Cambridge University Press, Cambridge 1986

[K] Kuhlmann, S.: Ordered Exponential Fields, The Fields Institute Monograph Series, vol. 12, AMS Publications (2000)

[KF] Kuhlmann, F.- V.: Dense subfields of Henselian fields, complements of valuation rings and integer parts, preprint (2004)

[M] Moniri, M.: Recursive models of open induction of prescribed finite transcendence degree with cofinal twin primes, C. R. Acad. Sci. Paris, 319 (1994), 903-908 
[M-R] Mourgues, M. - H. - Ressayre, J.- P. : Integer parts Every real closed field has an Integer Part, Journal of Symbolic Logic, 58 (1993), 641-647

[P] Pitteloud, D. : Existence of prime elements in rings of generalized power series, Journal of Symbolic Logic, 66 (2001), 1206-1216

[PC] Prieß-Crampe, S.: Angeordnete Strukturen. Gruppen, Körper, projektive Ebenen, Ergebnisse der Mathematik und ihrer Grenzgebiete 98, Springer (1983)

[R] Ressayre, J.-P.: Integer parts of real closed exponential fields, in: eds. P. Clote and J. Krajicek, Arithmetic, Proof Theory and Computational Complexity, Oxford University Press, New York (1993)

[Ri] Ribenboim, P.:Théorie des valuations, Les presses de l'université de Montréal, Montréal (1968)

[S] Shepherdson, J. C. : A Non-Standard Model for a Free Variable Fragment of Number Theory, Bulletin De L'Academie Polonaise Des Sciences, vol. XII, (1964)

Biljakovic:

Faculty of Agriculture, University of Zagreb, Croatia.

email: biljakovic@agr.hr

Kochetov:

Department of Mathematics, Carleton University, Ottawa, Canada.

email: kochetov@math.carleton.ca

Kuhlmann:

Research Unit Algebra and Logic, University of Saskatchewan, Canada.

email: skuhlman@math.usask.ca 